\title{
Una mirada a la estimulación temprana en el leguaje
}

\author{
A look at early stimulation in the language
}

Um olhar sobre a estimulação precoce na língua

Anny Gabriela Calle-Poveda I

gabriela850110@hotmail.com

Recibido: 18 de mayo de 2019 *Aceptado: 20 de febrero de $2019 *$ Publicado: 05 de abril de 2019

I. Magíster en Desarrollo Temprano y Educación Infantil, Licenciada en la Especialización de Terapia Ocupacional, Docente de la Universidad Laica Eloy Alfaro de Manabí, Manta, Ecuador. 


\title{
Resumen
}

La estimulación e intervención temprana constituyen un binomio esencial del trabajo preventivo desde la primera infancia, con un papel protagónico de las familias, orientadas y guiadas por los especialistas, colaboradores, promotores y ejecutores de la estimulación temprana. Se realizó una revisión de la literatura referente a la temática, se expuso las etapas por las que cursa el desarrollo normal del lenguaje, la estimulación temprana del desarrollo del lenguaje desde la visión Ciencia, Tecnología y Sociedad y se relacionó algunos ejercicios para la estimulación del lenguaje.

Palabras claves: Estimulación; desarrollo del lenguaje.

\begin{abstract}
The stimulation and early intervention constitute an essential binomial of preventive work from early childhood, with a leading role for families, guided and guided by specialists, collaborators, promoters and executors of early stimulation. A review of the literature referring to the subject was carried out, the stages through which the normal development of language was presented, the early stimulation of language development from the Science, Technology and Society vision and some exercises to stimulate the development of the language were related. language.
\end{abstract}

Keywords: Stimulation; language development.

\section{Resumo}

O estímulo e a intervenção precoce constituem um binômio essencial do trabalho preventivo desde a primeira infância, com papel protagonista para as famílias, orientadas e orientadas por especialistas, colaboradores, promotores e executores da estimulação precoce. Realizou-se uma revisão da literatura referente ao assunto, os estágios por meio dos quais foi apresentado o desenvolvimento normal da linguagem, a estimulação precoce do desenvolvimento da linguagem a partir da visão Ciência, Tecnologia e Sociedade e alguns exercícios para estimular o desenvolvimento da linguagem. idioma

Palavras-chave: Estimulação; desenvolvimento da linguagem.

\section{Introducción}

161 Vol. 5, núm. 2, abril, 2019, pp. 160-172

Anny Gabriela Calle Poveda 
Se conceptualiza la estimulación como un proceso natural que se pone en práctica en la relación diaria con el bebé, a través de éste el niño utilizará al máximo sus capacidades e irá ejerciendo mayor control sobre el mundo que le rodea al tiempo que sentirá gran satisfacción al descubrir que puede hacer las cosas por sí mismo.

A través de la repetición útil de diferentes eventos sensoriales que aumentan, por una parte, el control emocional proporcionando al niño una sensación de seguridad y goce, por otra amplían la habilidad mental que le facilita el aprendizaje, ya que se desarrollan destrezas, para estimularse a través del juego libre y del ejercicio de la curiosidad, la exploración y la imaginación, se desenvuelve la estimulación. Todo niño nace con la necesidad biológica de aprender y cualquier estimulación que se le brinde durante los primeros 12 meses, tiene más impacto en su crecimiento cerebral que en cualquier otra etapa de la vida.

Cabe agregar que el crecimiento total de un individuo se lleva a cabo por medio de la interrelación del desarrollo físico, mental, emocional y social, y la Estimulación Temprana producirá impacto en el crecimiento total del bebé, sin presionar ni acelerar ningún proceso de desarrollo. Simplemente, lo que busca es optimizar las capacidades del niño, en todas las áreas.

En ese mismo sentido la estimulación es muy sencilla de aplicar y que le va a proporcionar al niño las herramientas que necesita para el desarrollo de sus habilidades y un mejor desempeño en su futura etapa pre escolar. (González Zúñiga , 2007)

Como una alternativa para el adecuado desarrollo de las capacidades del niño se han instrumentado programas de estimulación temprana, entendiéndose por ésta a toda actividad que oportuna y acertadamente enriquece al niño en su desarrollo físico y psíquico.

La atención al desarrollo del lenguaje se realiza por parte de un especialista con competencias en su formación profesional para asumir la intervención desde edades tempranas. Se tiene en cuenta el enfoque comunicativo que prevalece en la investigación psicolingüística a partir de la década de los 80 y principios de los 90, la que ha puesto de relieve un factor importante en los mecanismos de formación del lenguaje: la comunicación como motor de la adquisición, el niño adquiere el lenguaje en entornos de comunicación. (Ygual \& Cervera, 1999) 
El desarrollo infantil es un proceso dinámico, sumamente complejo, que se sustenta en la evolución biológica, psicológica y social. (Narbona \& Chevrie-Muller, 2000), (López, 1998)

Dicha evolución va paralela al proceso de maduración del sistema nervioso que se inició desde su vida intrauterina y se caracteriza por la adquisición progresiva de funciones como el control de la postura, su autonomía al desplazarse, el vínculo con el medio circundante, la Comunicación y El Lenguaje Verbal, requiriendo de una adecuada vinculación entre su estructura genética, los factores biológicos y sociales. (Real Patronato de Prevención y de Atención a Personas con Minusvalía., 2000)

Existen dos elementos importantes que los niños conquistan en los primeros años de vida que son: el aprendizaje de la lengua materna junto con un rápido desarrollo psicomotor los cuales pueden verse afectado por la influencia de varios factores de riesgo. (Ríos , 2009), (Reyes Vargas \& Raymundo Molinero, 2000)

En la primera infancia aparecen la mayoría de las afecciones de la comunicación en el niño suceden, las dificultades del lenguaje y habla son las más prevalentes ; tienen impacto negativo en el desarrollo global del niño/a, tanto en sus relaciones sociales como en su vida afectiva, en el logro de habilidades cognitivas y, posteriormente en el aprendizaje escolar. (Schonhaut \& Maggiolo, 2012)

El desarrollo del lenguaje en el niño se realiza de forma ininterrumpida desde el nacimiento. Durante el primer año se forman las bases de la comunicación por medio de las interacciones que realiza con la familia y en las cuales son muy importantes la mímica facial, la entonación, la prosodia, el balbuceo, la coordinación sonido-vista, etc.; todo ello relacionado con el contexto y dentro de las denominadas funciones de comunicación. Además, durante esta época desarrolla la percepción auditiva y las habilidades fonológicas, y empieza a adquirir el lenguaje de su entorno, con aspectos específicos en el ámbito comunicativo y gramatical. Se produce un desarrollo muy importante entre los 2 y los 3 años. (López Salas \& Puebla Caballero, 2014)

Los presupuestos teórico-metodológicos del tema se enmarcan en los aportes de la escuela sociohistórico cultural de Vigotsky y sus seguidores, en relación con las categorías psicológicas: comunicación, actividad y personalidad; la unidad entre lo biológico, lo psicológico y lo social; lo afectivo y lo cognitivo; lo interno y lo externo; entre el pensamiento y el lenguaje en el desarrollo 
psíquico; entre la actividad y el psiquismo; el carácter activo, interactivo, sistémico y mediato de la psiquis humana y el método genético en la concepción del desarrollo. (Fernández Pérez )

En el marco del escenario anterior para Vigotsky Cito (...) "el período más saturado y rico en contenido, más denso y lleno de valor del desarrollo en general".... La regla fundamental del desarrollo infantil consiste en que el ritmo de desarrollo es máximo en el mismo inicio y por último la adquisición y desarrollo... depende en gran medida del medio social en el que vive el sujeto. Por tanto el hombre al nacer hereda toda la evolución filogénica, pero el resultado final de su desarrollo estará en correspondencia con las características del medio social en él que viva”. Vigotsky (1989: 2000).

\section{Etapas por las que cursa el desarrollo normal del lenguaje}

El desarrollo del lenguaje se da por etapas. Dichas etapas se cumplen en ciertos periodos de tiempo y edad. El lenguaje consta de cuatro aspectos: fonológico, sintáctico, semántico y pragmático. El aspecto semántico se refiere a la comprensión del lenguaje, el sintáctico a las estructuras del lenguaje para formar enunciados y proposiciones, el fonológico los fonemas o sonidos del habla y el pragmático al uso del lenguaje dentro de un contexto adecuado en situaciones comunicativas. (Cantwell \& Barker, 1978) Cantwell DP, Baker L. (1978 a). The language environment of autistic and dysphasic children. Journal of the American Academy of Child Psychiatry, 17: 604-613.

Existen varias clasificaciones para el desarrollo del lenguaje. Una de las más precisas y sencillas es la realizada por (Bloom, Lahey, \& Hood, 1980)

Esta clasificación identifica tres dimensiones del lenguaje: contenido, forma y uso; el contenido se refiere al significado o aspecto semántico, la forma se refiere a las palabras y a las relaciones que existen entre éstas y el uso tiene que ver con las funciones para las cuales habla el individuo y los cambios en su lenguaje de acuerdo con el contexto (pragmática).

\section{Etapa pre lingǘrstica (del nacimiento al año de edad)}

En esta etapa la comunicación se da mediante señas, gestos y ruidos. Las emisiones del niño no son reconocidas como palabras que emplean los adultos 
II. Etapa de una palabra (del año a los dos años de edad) Las emisiones del niño son reconocidas por los adultos como una palabra o aproximaciones a palabras empleadas por los adultos. Los adultos encuentran relación siempre entre la palabra que interpretan y la situación en la que el niño la produce.

III. Etapa del lenguaje telegráfico (del año y medio a los dos años seis meses) Sus emisiones son generalmente de dos o más palabras o aproximaciones. Sin embargo, aún no son reconocidas como oraciones. A pesar de que sus emisiones empiezan a ser más largas, no siempre son comprensibles, ya que emplea lo que comúnmente llamamos jerga, que se refiere al lenguaje que utilizan los niños cuando los padres hablan como si fueran de otro planeta. Emplea palabras de contenido (sustantivos y verbos) y no palabras de función (conectivos).

IV. Etapa de frases y oraciones simples (de dos años seis meses a tres años seis meses de edad) Sus emisiones son breves y algunas dependen del contexto, pero estas ya son reconocidas por el adulto como frases u oraciones. Existen funciones de sujeto y predicado.

V. Etapas de oraciones complejas (de los tres- cuatro años en adelante) Su comunicación verbal es más fluida; sus emisiones son más largas y libres de contexto. Sus oraciones complejas se forman de la unión de varias oraciones simples. Los avances posteriores son poco perceptibles. Desde el punto de vista fonológico, se observa que a la edad de tres a tres años y medio, los niños han adquirido los sonidos m, ch, ñ, k, t, y, p, n, l, f, y los diptongos ua y ue. Entre los cuatro y los cuatro años y medio, adquieren r, b, g, pl, bl, y el diptongo ie. Entre los cinco y los cinco años y medio, los niños de este estudio adquirieron los grupos kl, br, fl, kr, gr y los diptongos au y ei.

Por último, entre los seis y los seis años y medio, se observa la adquisición de los fonemas s, rr, de los grupos pr, gl, fr, tr y del diptongo eo. (Sur \& Rubenstein, 2005)

Debe concebirse el desarrollo del lenguaje como un proceso de aprendizaje socio fisiológico único e indivisible. (López Salas \& Puebla Caballero, 2014)

La estimulación temprana del desarrollo del lenguaje desde la visión Ciencia, Tecnología y Sociedad (CTS). 
El desarrollo infantil es un proceso dinámico, sumamente complejo, que se sustenta en la evolución biológica, psicológica y social. Los primeros años de vida constituyen una etapa de la existencia especialmente crítica, ya que en ella se van a configurar las habilidades perceptivas, motrices, cognitivas, lingüísticas y sociales que posibilitarán una equilibrada interacción con el mundo circundante. Ha sido estudiado como proceso por la Pediatría, la Neurología, la Psicología, la Pedagogía, la Lingüística y la Logopedia y Foniatría entre otras. (Real Patronato de Prevención y de Atención a Personas con Minusvalía., 2000)

El desarrollo del lenguaje es un proceso que ocurre en la familia fundamentalmente y en la sociedad en general, de esta forma el desarrollo de la personalidad presupone la asimilación de la experiencia social acumulada por muchas generaciones en la intercomunicación con los demás hombres en el proceso de enseñanza y educación

En este sentido la Psicología y la Pedagogía desde su surgimiento como ciencias trataron de dar respuesta a la demanda social de desarrollar hombres inteligentes. Desde los siglos XVII y XVIII comienzan estos estudios que toman al lenguaje como indicador importante. Se destacan los trabajos de precursores de la educación preescolar como J. A. Comenius, J. E. Pestalozzi, F. Fröebel, J. J. Rosseau, los cuales en el siglo XIX enfocaron este problema, en esta etapa surge la obra de Pestalozzi titulada "Guía para las madres sobre cómo enseñar a sus hijos a observar y hablar", en la que aparecen ideas acerca de la educación intelectual y tiene en cuenta el empleo de la lengua materna. (López Salas \& Puebla Caballero, 2014)

En este sentido la Psicología y la Pedagogía desde su surgimiento como ciencias trataron de dar respuesta a la demanda social de desarrollar hombres inteligentes. Desde los siglos XVII y XVIII comienzan estos estudios que toman al lenguaje como indicador importante. Se destacan los trabajos de precursores de la educación preescolar como J. A. Comenius, J. E. Pestalozzi, F. Fröebel, J. J. Rosseau, los cuales en el siglo XIX enfocaron este problema, en esta etapa surge la obra de Pestalozzi titulada "Guía para las madres sobre cómo enseñar a sus hijos a observar y hablar", en la que aparecen ideas acerca de la educación intelectual y tiene en cuenta el empleo de la lengua materna. (López Salas \& Puebla Caballero, 2014) 
Las obras de Fröebel y Pestalozzi aportan una serie de materiales autodidácticos, algunos especialmente elaborados para la estimulación del lenguaje. En el siglo XX se destacan las ideas de M. Montesori, Venguer, Piaget, Ausubel, L. S. Vigostki y los seguidores de sus teorías. (López Salas \& Puebla Caballero, 2014)

Muchos son los investigadores que han tratado el tema, pero es importante continuar abordando la estimulación como concepto en el manejo de menores en etapa preescolar para favorecer su desarrollo intelectual y específicamente el lenguaje.

Actualmente, se ha demostrado que los procedimientos propios de la Pedagogía y la Psicología, entre otras ciencias afines, son válidos para estimular, desarrollar y educar el lenguaje en correspondencia con las etapas de desarrollo en los niños/as y corregir, compensar o prevenir las alteraciones del lenguaje. En el caso de los trastornos de la comunicación oral es necesario corregirlos en el ambiente más normalizador y facilitador para el establecimiento de relaciones interpersonales y de interacción lingüística, el círculo infantil, la escuela primaria, la familia, la comunidad, como los ambientes que posibilitan al hombre la expresión de sentimientos, intereses, necesidades, afectos y a su vez son fuente de cognición y regulación de su propia actividad. (Fernández Pérez ,s.f)

En la contemporaneidad se aborda la estimulación como acciones dirigidas a la promoción en grupos sanos, no solo en riesgo o con alteraciones, con el fin de orientar a las familias en la conducción del desarrollo de sus hijos, facilitar la adquisición de los logros en cada etapa evolutiva, prevenir desviaciones y complicaciones.

La estimulación temprana como componente o parte esencial del trabajo preventivo se define como un sistema de acciones desarrolladoras de carácter integral que se concretan en un programa o guía de estimulación para potenciar y promover el desarrollo de los niños/as desde la primera infancia, tomando en cuenta su historia de vida, necesidades y demandas del desarrollo. (Fernández Pérez, 2005)

\section{Acciones dirigidas a la estimulación temprana del desarrollo del lenguaje.}

En el ambiente familiar el niño en edades temprana, aunque está lleno de cuidado y atención por parte del núcleo familiar, se ven disminuidas las posibilidades de tener un contacto directo y permanente 
con otros infantes de la misma edad y la implicancia que ello conlleva, no solo en el aspecto lúdico sino en su entorno general; ya que el lenguaje al ser una destreza que se ve influenciada por la calidad de la imitación fónica se desarrolla de mejor forma con la repetición de los sonidos, monemas y fonemas propios de esta etapa. Además muchos de los niños de la comunidades pertenecientes a las nacionalidades permanecen solos en el completo abandono desde edades tempranas debido a que el trabajo informal de los adultos basado en labores que en los que predomina el esfuerzo físico imposibilita la participación los mismos en estas labores agudizando cada vez su contacto lingüístico con otros seres humanos causando defíciencias en la articulación de la palabra y demás aspectos relacionados con el habla.

La estimulación del lenguaje oral en edades tempranas es muy importante, esto se justifica por la doble función del lenguaje para el ser humano, que por un lado nos sirve para comunicarnos y por otro como representación mental y estructuración del pensamiento, además permite prevenir y detectar problemas

El primer lenguaje que dominan los niños es la expresión de necesidades y sentimientos. Esto se logra mediante balbuceos, llantos y sonrisas en el transcurso de su desarrollo comienzan a nutrirse de palabras y frases para tales fines; así, conquistando un lenguaje más completo y fluido, llegando a un dominio total de la comunicación verbal.

Existen innumerables formas de estimular el desarrollo del lenguaje lo primero y más importante es hablarle y responderle siempre que intente comunicarse mientras que se realice esta comunicación de le debe mirar buscando centrar su atención.

Cada una de las actividades de estimulación temprana es la base primordial para que el niño y niña tenga un desarrollo óptimo en el área de lenguaje, mientras más pequeño se le estimule es mejor mediante canciones, cuentos, sonidos del medio por tal motivo desde que el bebé está en el vientre, en su nacimiento y en su desarrollo es bueno que se le estimule para que en un futuro no tenga problemas y pueda expresarse de la mejor manera con las personas que lo rodean.

\section{Relacionamos algunos ejercicios prácticos para la estimulación del lenguaje.}


$\checkmark$ Contando cuentos: A partir de los dos años en adelante leer un cuento a los niños les ayuda a que, poco a poco, comiencen a comprender aquello que se les está contando y, a su vez, estimular el habla. No es de preocupación que los niños no respondan rápidamente a la comprensión de los relatos, en esta etapa se le debe facilitar esta tarea relatando cuentos que sean acordes a su edad.

$\checkmark$ Cantando canciones: Enseñar canciones infantiles ayuda a que los niños no sólo estimulen su sentido del oído y atención, sino que también promueve la motricidad gruesa cuando dichas canciones indican que el niño debe acompañar la misma con movimiento de brazos, piernas, manos, cabeza, etc., a la vez que fomenta las relaciones de comunicación entre sus compañeros. Esto favorece el habla como medio de comunicación entre ellos.

$\checkmark$ Fotos o imágenes: ¿Qué estamos haciendo? Mostrarles fotos a los niños, favorece su interés por la comunicación. En estos casos se sugiere que las fotos sean de personas conocidas por ellos (familiares, por ejemplo) para que ellos puedan relatar qué es lo que la familia está haciendo en esa fotografía o bien, quiénes son los que aparecen en dicha foto. Tras realizar estas simples preguntas el niño comenzará a hablar y, poco a poco, irá enriqueciendo su lenguaje.

$\checkmark$ Los títeres o las marionetas: Las obras infantiles que usan marionetas o títeres favorecen el habla en los niños. En estos casos, sugerimos que los mismos personajes (títeres) interactúen con los niños. Esto llama poderosamente su atención y ayudará a que ellos se animen a hablar.

$\checkmark$ Utilizar la tecnología para ayudarles a los niños pequeños: grabadora de voz: La tecnología puede enseñarles rápidamente el tono de su propia voz, así como también la intensidad con la que el niño habla. De este modo no sólo se estimula el habla sino también la escucha, parte esencial para que los niños hablen del modo adecuado. (Estimulación del lenguaje: Actividades de lenguaje para niños, 2014)

La estimulación temprana mejorará las condiciones físicas, emocionales, cognitivas y sociales de los niños y niñas, potenciando la psicomotricidad, para elevar el rendimiento escolar, y los resultados de evaluación en todas las etapas educativas. El rol principal es de los padres de familia, ente fundamental en la educación integral y participación activa en la sociedad, además de que se requiere de una atención profesional especializada en esta área. Proceso que garantizará el desarrollo integral infantil 
y por ende se potenciará el desarrollo humano, mencionado en el Plan Nacional del Buen Vivir 20132017, como el conjunto de técnicas y condiciones que debe tener todo infante, porque posee efectos muy significativos, sobre todo en el desempeño escolar y en su formación personal. (Secretaria Nacional De Planificación y Desarrollo (SENPLADES), 2013)

Se concluye que el desarrollo del lenguaje en edades tempranas debe estar dirigido a acciones encaminadas a la preparación de las familias para enfrentar la estimulación temprana, como concepto a la promoción y prevención de alteraciones de la comunicación, así como la integración de las acciones gubernamentales, en aras de fortalecer el trabajo en la comunidad desde el servicio de Logo fonoaudiología.

\section{Referencias Bibliográficas}

Bloom, L., Lahey, M., \& Hood, L. (1980). Complex sentences: acquisition of syntactic connectives and the semantic relations they encode. J child lang., 7 (2): 235-61.

Cantwell, D. P., \& Barker, L. (1978). The language environment of autistic and dysphasic children. Journal of the American Academy of Child Psychiatry, 17: 604-613.

Estimulación del lenguaje: Actividades de lenguaje para niños. (2014). Estimulación del lenguaje: Actividades de lenguaje para niños. . Obtenido de https://www.educapeques.com/escuela-depadres/estimulacion-del-lenguaje-ninos.html

Fernández Pérez, A. G. (2005). Prevención y atención integral a niños con necesidades educativas especiales de edad temprana y preescolar. La Habana: Editorial Pueblo y Educación.

Fernández Pérez, A. G. (s.f.). Enfoque cubano de atención a los trastornos de la comunicación y el lenguaje Actualidad y perspectivas. Obtenido de http://www.sld.cu/galerias/pdf/sitios/rehabilitacionlogo/enfoque_cubano_de_atencion_a_los_trastornos_de_la_comunicacion_y_el_lenguaje._a ctualidad_y_perspectivas.pdf 
González Zúñiga, G. C. (2007). Los programas de estimulación temprana Desde la perspectiva del maestro. $\quad$ LIBERABIT; 13(3). Obtenido de http://www.scielo.org.pe/pdf/liber/v13n13/a03v13n13.pdf

López, B. M. (1998). El lenguaje del niño. La Habana: Editorial Ciencia y Técnica.

López Salas, D. M., \& Puebla Caballero, N. M. (2014). Acciones intersectoriales en la estimulación temprana del desarrollo del lenguaje. Humanidades Médicas, 14(3), 659-675.

Narbona, J., \& Chevrie-Muller. (2000). El lenguaje del niño. Desarrollo normal, evaluación y trastornos. Barcelona: Editorial Masson.

Real Patronato de Prevención y de Atención a Personas con Minusvalía. (2000). Grupo de Atención Temprana. Libro blanco de la atención temprana del Centro Español de Documentación sobre Discapacidad. Madrid. Madrid: Editorial ARTEGRAF.

Reyes Vargas, N., \& Raymundo Molinero, M. (2000). Evaluación del neuro desarrollo a los dos años de vida en recién nacidos menores de 2000 gramos incluidos en el programa madre canguro del hospital escuela. Rev Med Post UNAH. 5(2). Obtenido de http://www.bvs.hn/RMP/pdf/2000/pdf/Vol5-2-2000-10.pdf

Ríos, I. (2009). Transformaciones en la concepción curricular de la Educación Preescolar cubana. MINED.

Schonhaut, B. L., \& Maggiolo, L. M. (2012). Alteraciones del lenguaje en la población preescolar. Otorrinolaringología. Suplemento de Salud (i) Ciencia. Vol.7, N04 Julio.

Secretaria Nacional De Planificación y Desarrollo (SENPLADES). (2013). Plan Nacional Del Buen Vivir 2013-2017 .República Ecuador.

Sur, M., \& Rubenstein, J. M. (2005) Patterning and plasticity of the cerebral cortex. Science; 310:805.

Ygual, A., \& Cervera, J. F. (1999). Intervención logopedia en los trastornos de la adquisición del lenguaje. Rev Neurol. 28 (Supla 2):109-18. Obtenido de 
http://www.sld.cu/galerias/pdf/sitios/rehabilitacion-

logo/intervencion_logopedica_en_los_retrasos_del_lenguaje.pdf 